\title{
Calcification patterns in femoral and carotid atheromatous plaques: A comparative morphometric study
}

\author{
MIRCEA CATALIN COSARCA ${ }^{1 *}$, EMŐKE HORVÁTH $^{2 *}$, CALIN MOLNAR $^{3}$, \\ GYOPÁR-BEÁTA MOLNÁR ${ }^{4}$, ELIZA RUSSU ${ }^{5}$ and VASILE ADRIAN MUREȘAN ${ }^{5}$
}

${ }^{1}$ Doctoral School and Departments of ${ }^{2}$ Pathology and ${ }^{3}$ General Surgery, 'George Emil Palade' University of Medicine, Pharmacy, Science and Technology, 540142 Târgu-Mureș; ${ }^{4}$ Pathological Anatomy Service, Târgu-Mureș County Emergency Clinical Hospital (SCJU Târgu-Mureș), 540136 Târgu-Mureș;

${ }^{5}$ Department of Vascular Surgery, 'George Emil Palade' University of Medicine, Pharmacy, Science and Technology, 540142 Târgu Mureș, Romania

Received April 5, 2021; Accepted May 5, 2021

DOI: $10.3892 / \mathrm{etm} .2021 .10297$

\begin{abstract}
This comparative study was designed to focus on the mineral patterns in human atherosclerotic plaques based on quantitative measurements of calcium deposits through the morphometric method. A total of 101 atherosclerotic plaques were harvested by conventional transluminal angioplasty from the carotid artery (CA) and different segments of the femoral-popliteal axis (FPA), fixed in formalin and sent for histological processing. The histological grade of the atherosclerotic plaque and the calcification pattern were evaluated, followed by a morphometric analysis of the mineral deposits. Regarding the localization, the advanced plaques (VII and VIII types) developed predominantly at the level of the superficial femoral artery (SFA) compared to the CA $(\mathrm{P}<0.001)$. This significant difference was maintained even if they were divided into low grade (IV and V) and high grade categories (VI, VII and VIII) $(\mathrm{P}<0.05)$. Compared with that in the carotid plaques, in the FPA plaques the mineralized surface increased in parallel with the narrowing of the vascular lumen diameter. The image analysis of the total pathological calcification score (pCS) showed a significant difference between the CA plaques and distal SFA (dSFA) plaques $(\mathrm{P}=0.038)$ and between the proximal SFA (pSFA) and dSFA plaques $(\mathrm{P}=0.013)$. In the case of the simple nodular pattern, calcification occupied significantly larger areas in the plaques developed in the dSFA and popliteal artery (PA) in comparison with the CA plaques
\end{abstract}

Correspondence to: Dr Emőke Horváth, Department of Pathology, 'George Emil Palade' University of Medicine, Pharmacy, Science and Technology, 38 Gh. Marinescu Street, 540142 Târgu-Mureș, Romania

E-mail: emoke.horvath@umfst.ro

*Contributed equally

Key words: calcification, atherosclerosis, thromboendarterectomy, digital morphometry, peripheral arterial disease
$(\mathrm{P}=0.0007$ and $\mathrm{P}=0.0009)$. pCSs calculated in plaques with extensive calcification pattern showed a lower value in the $\mathrm{CA}$ vs. the pSFA plaques $(\mathrm{P}=0.004)$. A less pronounced, but significant difference was observed between the pCS of pSFA and dSFA plaques $(\mathrm{P}=0.017)$. Femoral and carotid plaques exhibited different morphology and tendency for calcification. In parallel with the narrowing of the vascular lumen diameter, the mineralized surface increased at the level of different FPA segments. These results suggest that the mechanism is site-specific, and wall structure-dependent.

\section{Introduction}

Circulatory diseases, the leading causes of death among the elderly in the EU, are major public health concerns that contribute to a burden on health care services; the main diseases being ischemic heart disease and ischemic cerebrovascular disease (1). Behind these diseases is usually atherosclerosis, developed following a complex pathophysiological process leading to endothelial dysfunction, intimal thickening, inflammation and vascular calcification $(2,3)$. Some studies have demonstrated that the prevalence of acute coronary events is higher in patients with peripheral arterial disease (PAD) than in those without PAD; therefore, PAD can be considered as a coronary artery disease equivalent (4-6). Coronary and carotid artery atherosclerosis have been extensively investigated in several studies that have focused on the role of calcification in plaque instability pushing the involvement of the femoral-popliteal axis (FPA) in the background, despite the clinical importance of atherosclerosis at its level; calcification in the lower extremity is considered the iceberg of cardiovascular disorders, which in diabetes or chronic kidney disease doubles the risk of cardiovascular mortality and quadruples the risk of amputation, the most dreaded complication $(4,7)$. Vascular calcification, the major complication of atherosclerosis, results from the deposition of calcium phosphate salt in the form of hydroxyapatite in the plaque and arterial wall. Yet, the etiology of calcification remains unclear and is an area of active research. Although previous analyses have associated 'macrocalcification' with plaque stability $(8,9)$, recent studies have suggested that superficial and multiple calcifications and 
ulceration are associated with intra-plaque hemorrhage, and they may be a substitute for higher risk lesions $(10,11)$. Some studies have investigated the role of size and location of calcification regarding the restenosis rate after surgical treatment. Compared with the low percentage of restenosis after coronary drug-eluted stent replacement (10-15\%) (12), restenosis after lower limb artery stenting reaches $40-50 \%(13,14)$, while the variable rate of this complication is between 1 and $36 \%$ after intervention at the carotid level (15).

Numerous factors such as demography, comorbidity, sex, cardiovascular risk factors, high-degree stenosis, metabolic and hemodynamic factors, biochemical parameters, the lesion length, the underlying site-specific wall structure and the nature (including calcification) of the atheromatous plaque alter the restenosis rate after endarterectomy or stent implantation, of which the crucial role is attributed to the appearance and extent of calcification $(16,17)$. Atherosclerotic plaque calcification varies according to each arterial bed; femoral plaques exhibit a significantly different type of calcification pattern and osteoid metaplasia than carotid plaques (18), which suggests the role of a site-dependent mechanism in plaque calcification. Clinically, two types of plaque calcification have distinct implications in the progression and regression of atherosclerosis: Macrocalcification, which leads to plaque stability, and microcalcification with a pivotal role in plaque rupture and consequent complications $(19,20)$. Recent research has demonstrated that the spatial distribution of mineral mass, calcification size and location are important determinants of the plaque rupture risk $(21,22)$.

The effect of calcification is considered biphasic, from pro-inflammatory properties of 'microcalcification' to anti-inflammatory properties of 'macrocalcification'. Plaque rupture has been shown to be positively correlated with microcalcifications, and conversely with extensive calcifications. Macroscopic calcification is easily detected and quantified (calcium scores as predictive value for cardiovascular incidence) using the CT scan method. In contrast, microcalcification, the early stage of plaque calcification, is observed only with positron emission tomography (PET)/CT imaging and optical coherence tomography (diagnostic methods that are not used in daily practice) (9).

However, the CT analysis of calcification patterns is limited by the resolution and blooming artefacts. In this context, the histopathological examination of the endarterectomy specimens provides useful information to the clinician to develop a treatment strategy.

This present study is a detailed cross-sectional morphological comparative characterization of the intra-plaque calcification of advanced atherosclerotic lesions based on morphometric methods involving the most important segments of the FPA and CA.

\section{Materials and methods}

Patients and tissue fragments. In this prospective comparative study, tissue fragments harvested by conventional transluminal angioplasty from patients diagnosed with symptomatic PAD and CA atherosclerosis were included. The material was collected from different patients between January 2017 and December 2018, at the Vascular Surgery Clinic, within the Mureș County Emergency Clinical Hospital (Romania).
A total of 101 cases were selected for the clinic pathological study, based on strict criteria which included patients with complete clinical documentation and the written consent of enrolment in the study and an appropriate quantitative and structural specimen for histological examination. Prior to surgery, for the clinical assessment of the severity of the CA and the FPA stenosis, a CT angiography and a Doppler ultrasonography were performed. The subjects were first divided into two groups: Group $1(n=21)$ included patients with symptomatic CA stenosis (either transient ischemic attack or stroke on the ipsilateral side of the carotid stenosis); and group $2(n=80)$, patients with stenosis in different segments of the FPA presenting claudication or critical limb ischemia. The atherosclerotic plaques from the FPA were collected from the following levels: The proximal third of the superficial femoral artery (pSFA), adjacent to the inguinal ligament (Poupart's ligament); the distal segment of the superficial femoral artery (dSFA) at the level of the Hunter's canal and the proximal segment of the popliteal artery (PA), respectively. All samples from the carotid artery (CA) and FPA were immediately fixed in $10 \%$ neutral buffered formalin and sent for histological processing. The study was conducted according to the principles of the Helsinki Declaration and was approved by the Ethics Committee of the 'George Emil Palade' University of Medicine, Pharmacy, Science and Technology of Târgu-Mures,, Romania (no. 884 and 11420/30.04.2020). All patients signed an informed consent for inclusion in the study.

Histological processing. The specimens were processed using a standard method to produce paraffin sections for staining with hematoxylin and eosin (H\&E), and the calcified specimens were treated with an ethylenediaminetetraacetic acid (EDTA) solution (pH 7.0).

Establishment of the histological grade of the atherosclerotic plaques. The histological grade of the atherosclerotic plaque was evaluated on $4-\mu \mathrm{m}$ thick and H\&E-stained sections according to the modified American Heart Association (AHA) classification based on 9, well-defined categories, of which 6 types correspond to atheromatous plaque (23).

Briefly, type IV is considered to be the first advanced stage of the disease with confluent extracellular lipid core. An atheromatous plaque with fibrous cap associated with prominent fibrosis is included in type V. All atheromatous plaques with surface damage (ulceration and thrombosis) must be classified as type VI. A fibrocalcified plaque with extensive calcification is categorized as type VII. In type VIII, fibrous changes predominate. Complete occlusion (type IX) in our study was not taken into account.

Determination of the degree (type) of calcification of the plaques. Focusing on the calcification pattern, the plaques were included in four categories depending on the calcified patch distribution, size and shape: a) Sheet-like calcification (defined as numerous micronodules/scattered small mineral foci forming a calcification front within fibrosis (Fig. 1A); b) nodular calcification (single/multiple stratified mineral deposits with a nodular aspect (Fig. 1B); c) extensive (confluent) calcification (conglomerate of mineral material with irregular edges (Fig. 1C); and d) osteoid metaplasia (mature bone with 

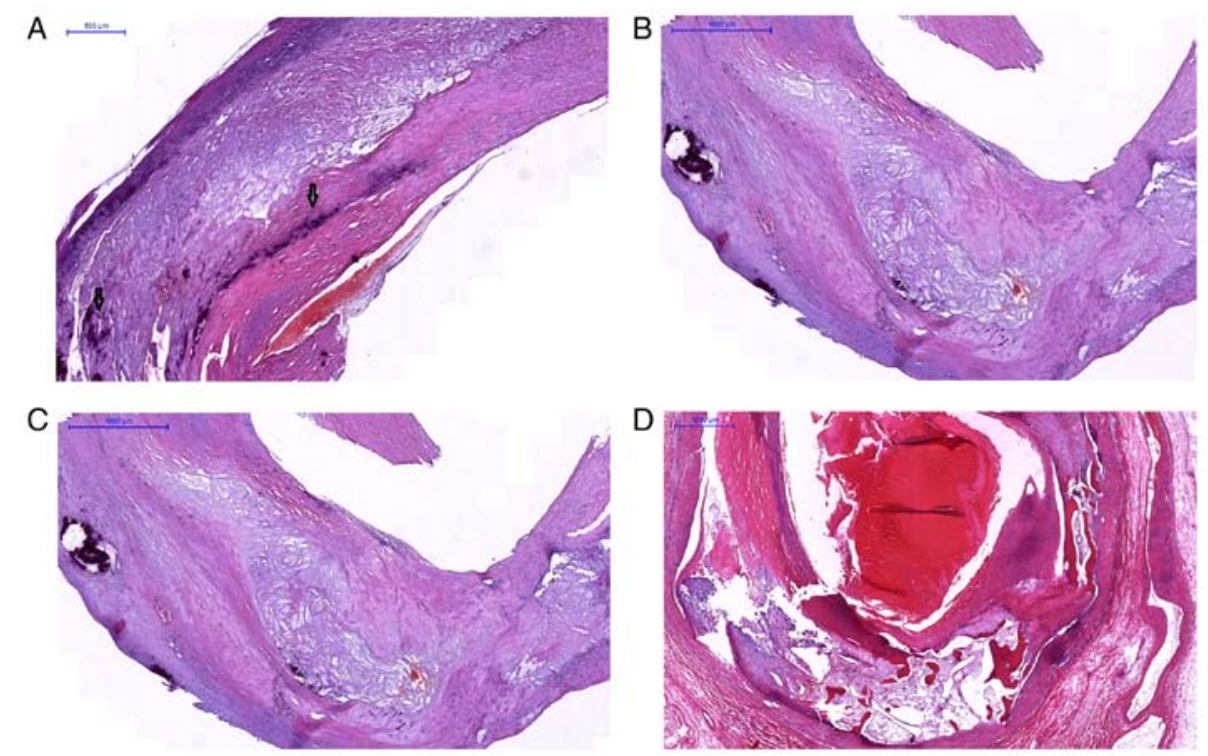

Figure 1. Microscopic aspects of human atherosclerotic plaque calcification types; H\&E staining. (A) Sheet-like calcification (defined as numerous micronodules/scattered small mineral foci forming a calcification front within fibrosis. (B) Nodular calcification (single/multiple stratified mineral deposits with nodular aspect. (C) Extensive (confluent) calcification (conglomerate of mineral material with irregular edges and disruption of internal elastic lamina and (D) osteoid metaplasia represented by mature bone with lamellar structure and bone marrow. H\&E, hematoxylin and eosin.

lamellar structure and bone marrow (Fig. 1D). Although there is no conventional standard of size, there is a general consensus that categorizes microcalcifications and macrocalcifications based on nodules of $<50$ and $\geq 50 \mu \mathrm{m}$, respectively (19).

The site-specific prevalence for each type of calcification was compared. The comparative study of the histology of atherosclerotic plaque and degree of calcification depending on the location (carotid and femoral-popliteal axis) was observed by digital morphometry. The selected calcified plaques (81 cases) were grouped according to the involved arterial segments (CA, pSFA, dSFA and PA). Their associated H\&E stained slides were digitally scanned at x20 magnification with a Mirax Scanner and were examined with the associated Panoramic Viewer 1.15.4 software (3DHISTECH Ltd., Budapest, Hungary). A comparative microscopic examination was performed by the parallel evaluation of the plaque thickness (using $\mathrm{x} 4$ magnification) at the CA and the pSFA.

Morphometric analysis of mineral deposits. In order to quantifying the total calcified (positive) surface area (\%) of the atheromatous plaques in the two locations mentioned above, the scanned histological sections were captured with objective 4, saved in Tiff format, transferred to the NIH's Image J 1.46 program (National Institutes of Health, USA) and followed by processing of the obtained image using HSB (hue, saturation, brightness) color filtering (24). Depending on the size and extent of calcification, 3 to 5 different representative locations were selected along each tissue. Calcified patches were tagged as positive surface; the percentage of the total examined area noted as the pathological calcium score (pCS) (Fig. 2).

Statistical analysis. The descriptive and comparative statistics were performed with GraphPad Prism 8 (GraphPad Software, Inc.). Multiple groups were compared through the Kruskal-Wallis ANOVA test, and group comparisons (involved arterial segment and pCS) were made with the non-parametric
Mann-Whitney U test. A correlation analysis was performed according to Spearman. The level of statistical significance was set at $\mathrm{P}<0.05$.

\section{Results}

Patient characteristics. During the aforementioned period, 138 patients were diagnosed with PAD, of which 101 met the criteria for enrollment in the study. The mean age of the patients was $66.35 \pm 8.31$ (range $48-85$ ), $45.54 \%$ of the cases being included in the age category $60-69$ years. The distribution of patients by sex showed a male predominance $(77.2 \%$ vs. $22.8 \%)$ with a significant difference $(\mathrm{P}<0.001)$ between the two sexes.

Among all patients, $67.33 \%$ had claudication and $32.67 \%$ had acute limb-ischemia. When reviewing the cardiovascular risk factors, $75.24 \%$ had hypertension, $29.7 \%$ had diabetes, $78.21 \%$ had hyperlipidemia, $62.37 \%$ had a history of tobacco use, and $32.67 \%$ were obese (body mass index $>30.0$ ). A total of $66 \%$ of the hypertensive patients showed an atherosclerosis of the FPA. There was no significant difference $(P>0.05)$ between the sexes in terms of cardiovascular risk factors.

Anatomical location of the stenosis. Based on the imaging examinations (CT angiography and Doppler ultrasonography) results, we classified the cases dependent on the narrowed arterial segment in four categories: I, 21 CA plaques (20.8\%); II, 23 plaques from the pSFA (22.77\%); III, 46 plaques from the dSFA (45.54\%); and IV, 11 PA plaques (10.89\%).

Establishment of the histological grade of the atherosclerotic plaques in the different arterial segments. According to the modified AHA classification criteria, a large part of the atherosclerotic plaques were classified based on the morphological aspect in type VII and VIII (70 cases). 

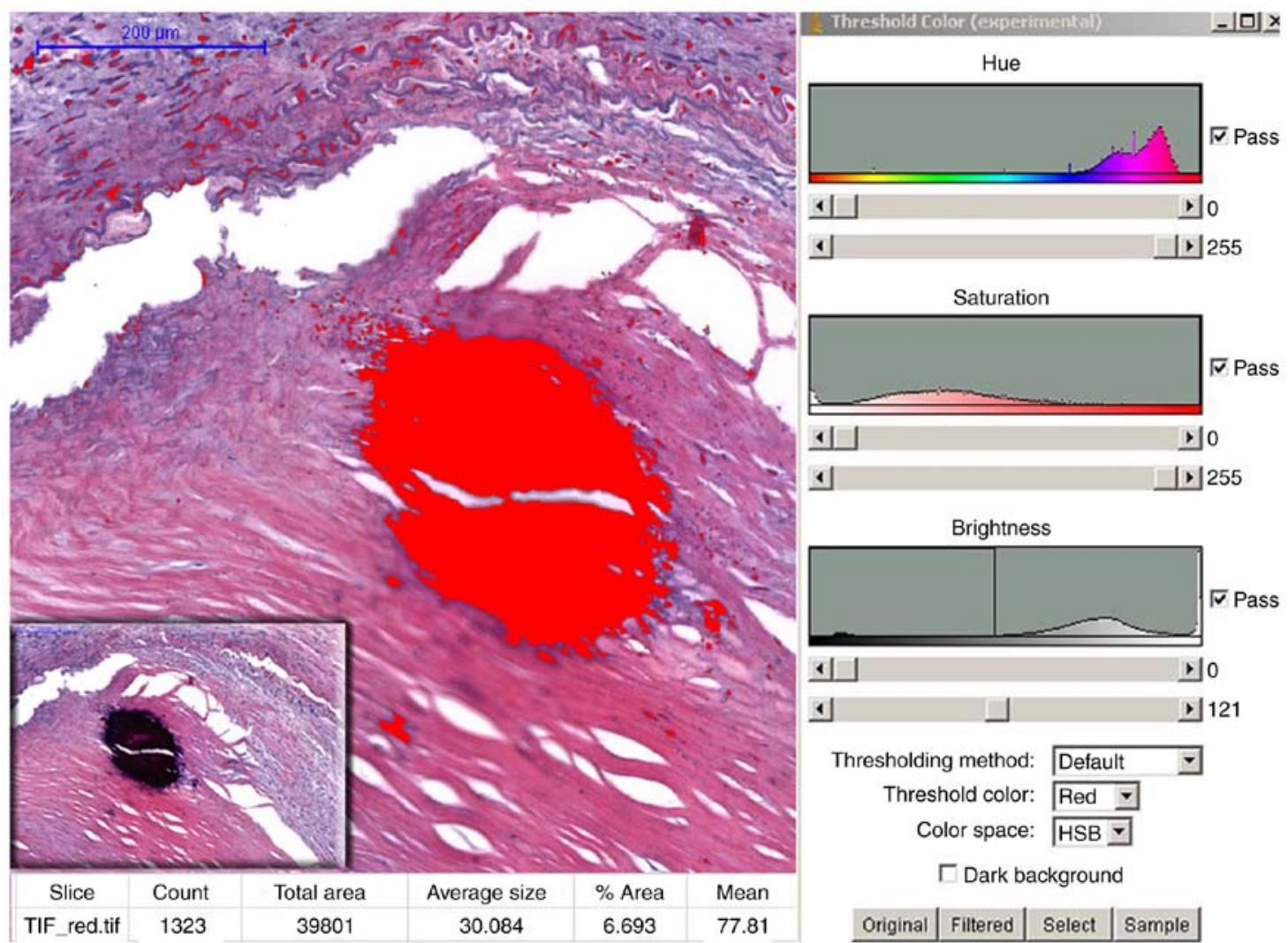

Figure 2. Morphometric analysis of mineral deposits for the quantification of the pCS (\% area) in the atheromatous plaques. Representative foci with calcification (H\&E stain, inset) were captured with x 20 magnification, followed by processing of the obtained image and HSB (hue, saturation, brightness) color filtering of the NIH's Image J 1.46 software. H\&E, hematoxylin and eosin.

Regarding the localization, we found that the plaques included in AHA VIII type (45 cases) developed predominantly at the level of the superficial femoral artery [proximal third and the distal segment (62.5\%) compared to the CA $(23.8 \%)(\mathrm{P}<0.001)]$. This significant difference was maintained even when they were divided into low grade (AHA IV, V) and high grade categories (AHA VI, VII and VIII) $(\mathrm{P}<0.05)$.

Determination of the degree (type) of calcification of the plaques. Of the 101 cases, only 20 did not show calcifications in the examined plaques. Regarding the degree of intra-plaque calcification, 27 cases were classified as subtype I (sheet-like calcification); 32 cases were classified as subtype II, simple nodular calcification; 20 cases were classified as subtype III with extensive calcification; and 2 cases were categorized as subtype IV with osteoid metaplasia. Most cases of plaque calcifications $(\mathrm{n}=36)$ were from the FPA $(44.45 \%)$, of which 17 (47.23\%) fell into subtype II (nodular calcification) (Fig. 3). Statistical analysis indicated a significant difference between CA and FPA plaques in terms of nodular calcification (type II) in favor of FPA $(\mathrm{P}<0.05)$.

The morphometric analysis of plaque thickness in carotid and femoral endarterectomy specimens did not show significant differences in the average thickness of the plaques at the level of the pSFA $(2,000.67 \pm 202.98 \mu \mathrm{m}$; range $1,680.15$ to $2,462.3 \mu \mathrm{m})$ compared to CA $(1,977.17 \pm 231.88 \mu \mathrm{m}$; range $1,654.34$ to $2,424.15 \mu \mathrm{m})(\mathrm{P}<0.05)$. This mild variation was also underlined by the values of the ratio plaque thickness/total thickness in the two mentioned locations (0.89 vs. 0.87) (Fig. 4).
Comparative morphometric analysis of mineral deposits in the femoral and carotid artery plaques. In the present study, we included only calcified plaques (from 81 cases), that showed a higher frequency among men $(\mathrm{P}<0.001)$. Image analysis of the total mineralized area of atherosclerotic plaques from the four locations showed different results concerning the amount of deposited mineral salts. Ignoring the calcification subtype, there was no significant difference between the pCS of pSFA (13.55 \pm 9.58$)$ and the CA plaques $(14.84 .55 \pm 10.96)(\mathrm{P}=0.704)$. In contrast, with the decrease in the vascular caliber, a statistically significant difference appeared between the pCS of the CA plaques $(14.84 .55 \pm 10.96)$ and $\mathrm{dSFA}(21.63 \pm 11.39)$ plaques $(\mathrm{P}=0.038)$, respectively the pSFA $(13.55 \pm 9.58)$ and dSFA $(21.63 \pm 11.39)$ plaques $(\mathrm{P}=0.013)$ and the pSFA $(13.55 \pm 9.58)$ and PA $(22.47 \pm 12.27)(\mathrm{P}=0.048)$. Another noteworthy result was the increase in the mineralized surface at the level of the different FPA plaque segments, in parallel with the narrowing of the vascular lumen diameter.

We compared the calcified area of the plaques developed in the above segments also according to the subtype of calcification. Regarding the sheet-like plaque calcification pattern in the paired comparison of the FPA segments (pSFA vs. dSFA, pSFA vs. $\mathrm{PA}$ ), we found no significant difference ( $\mathrm{P}=0.134$ and $\mathrm{P}=0.49$ ). Because in the CA plaques this pattern developed in only two cases, this segment was not included in the paired comparison. The simple nodular calcification pattern did not show significant differences between the pCS of the CA and the pSFA plaques $(\mathrm{P}=0.06)$. In contrast, $\mathrm{pCSs}$ of the $\mathrm{CA}$ were much lower than those in dSFA and PA, respectively, segments 


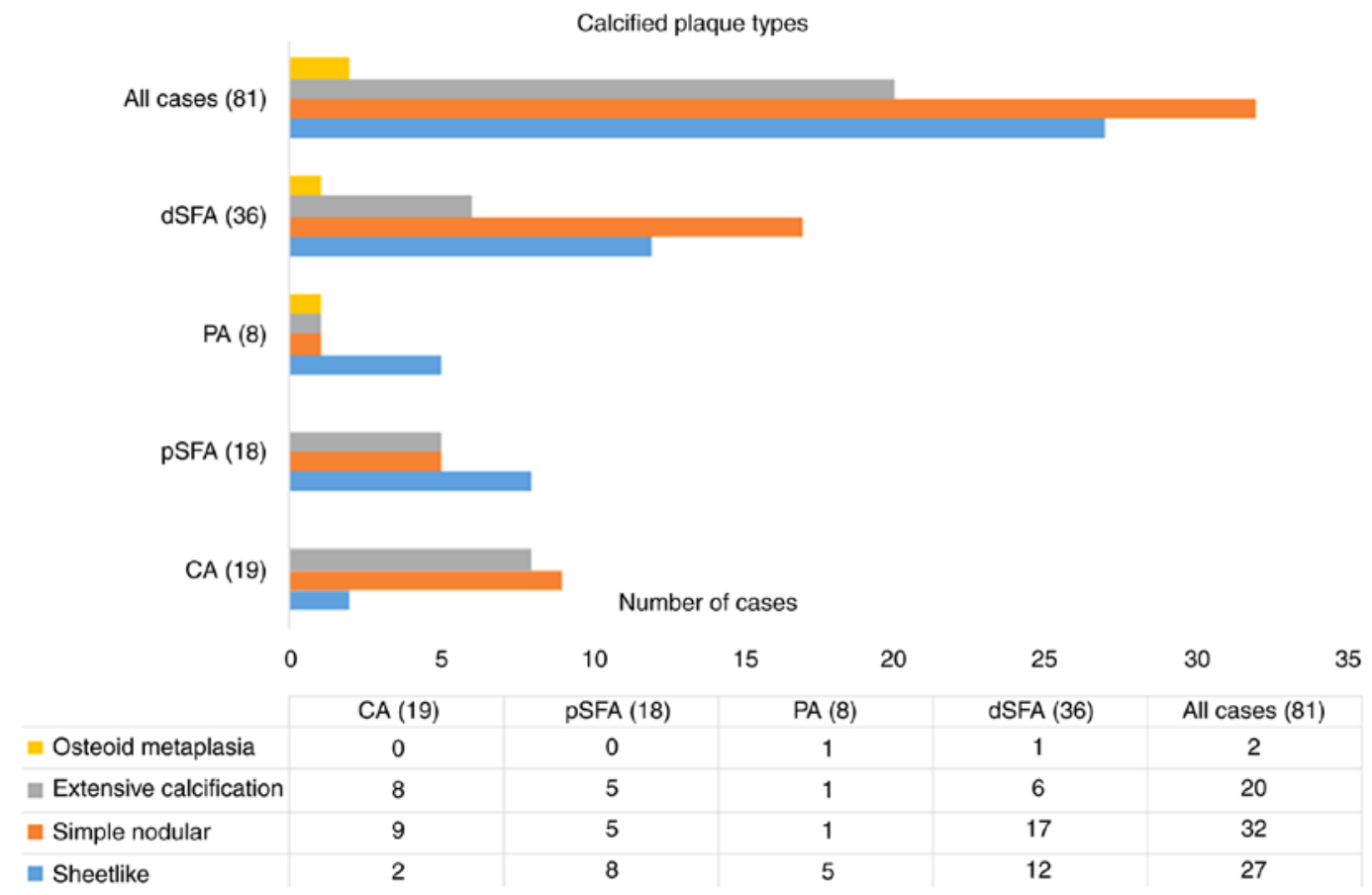

Figure 3. Distribution of plaques according to the calcification type in different arterial segments. Statistical analysis shows a significantly higher presence of nodular calcification (type II) in the femoral-popliteal axis (FPA), than in the carotid arterial (CA) bed. dSFA, distal segment of the superficial femoral artery; pSFA, proximal third of the superficial femoral artery; PA, popliteal artery.
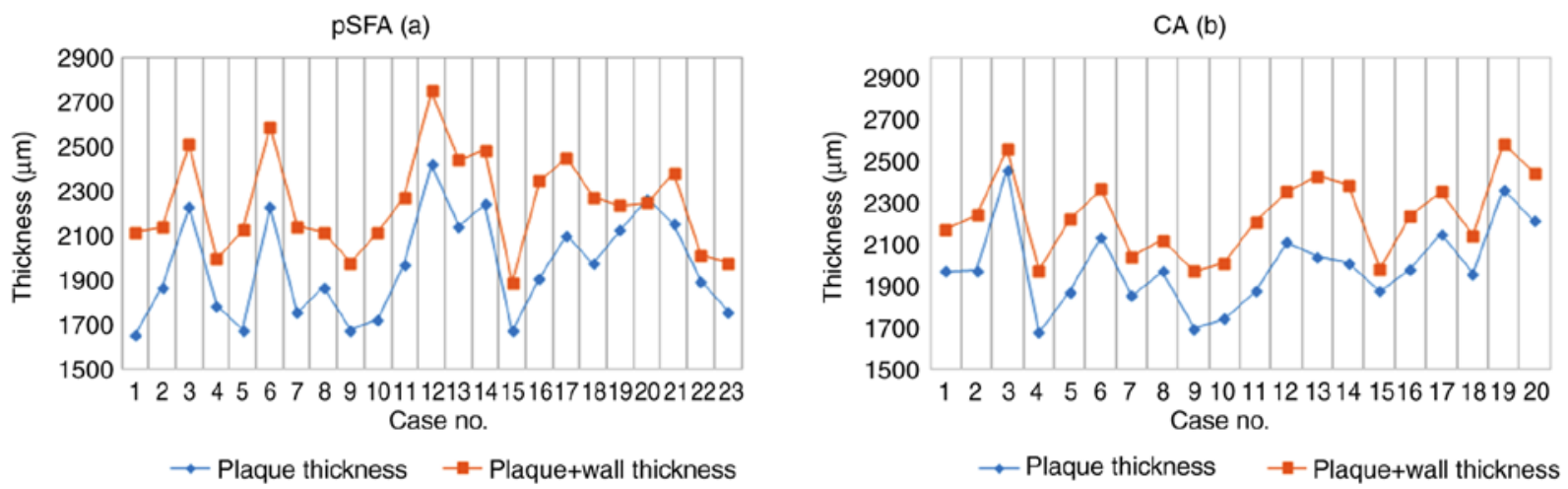

Figure 4. The average thickness of the plaques and the values of the ratio plaque thickness/total thickness at the level of the pSFA (a) compared to CA (b) did not show a significant difference. pSFA, proximal third of the superficial femoral artery; CA, carotid artery.

at which calcification occupied significantly larger areas $(\mathrm{P}=0.0007$ and $\mathrm{P}=0.0009)$. At the level of pSFA segments, the nodular calcification showed significant differences in favor of the PA $(\mathrm{P}=0.007)$. Extensive calcification surface area did not differ between the CA and pSFA plaques $(\mathrm{P}=0.83)$, but the pCS of the CA plaques was lower than those measured in dSFA $(\mathrm{P}=0.004)$. A less pronounced, but significant difference was observed between the pCS of the pSFA and dSFA plaques $(\mathrm{P}=0.017)$ (Table I and Fig. 5).

\section{Discussion}

The majority of studies focusing on calcification are based on calcium score (CS) determination by imaging; few of them use morphometric analysis of mineral content on the histopathological sections of the endarterectomy samples involving one type of artery (21) or two types (18).
According to our knowledge, this comparative study between the carotid artery (CA) and the femoral-popliteal axis (FPA) is the first to follow the extent of calcification depending on the variation of the vascular caliber.

Our patients undergoing carotid and femoral endarterectomy represented a select risk group with high grade artery stenosis; a large part of the atherosclerotic plaques being classified based on morphological aspects of type VII and VIII, over three quarters of them with intra-plaque calcification. Preliminary histological studies demonstrated considerable differences between plaques with identical degrees of stenosis, respectively. Certain plaque features are associated with an increased risk of ischemic event (25). At the same time, it has been shown that carotid plaque thickness or plaque volume is more strongly associated with ischemic events than the degree of stenosis (26). Although vascular calcification in the lower extremities is commonly a hallmark of peripheral 
Table I. Comparison of total calcified area in different arterial segments.

Arterial segment

\begin{tabular}{lcccc}
\cline { 2 - 5 } Total calcified area $(\mathrm{pCS})$ & CA plaque $(\mathrm{n}=19)$ & pSFA plaque $(\mathrm{n}=18)$ & dSFA plaque $(\mathrm{n}=35)$ & PA plaque $(\mathrm{n}=9)$ \\
\hline I. Sheet-like calcification & $2.09 \pm 0.47$ & $6.34 \pm 0.85$ & $8.27 \pm 0.75$ & $8.07 \pm 1.25$ \\
II. Nodular calcification & $7.5 \pm 1.06^{\mathrm{e}, \mathrm{f}}$ & $11.27 \pm 0.88^{\mathrm{d}, \mathrm{g}}$ & $26.09 \pm 1.47^{\mathrm{e}, \mathrm{g}}$ & $27.89 \pm 2.90^{\mathrm{d}, \mathrm{f}}$ \\
III. Extensive calcification & $26.28 \pm 2.00^{\mathrm{c}}$ & $27.33 \pm 2.46^{\mathrm{b}}$ & $35.73 \pm 1.59^{\mathrm{b}, \mathrm{c}}$ & $38.52 \pm 0.00$ \\
Average calcification & $14.88 \pm 2.51^{\mathrm{a}}$ & $13.54 \pm 2.26^{\mathrm{b}}$ & $21.63 \pm 1.93^{\mathrm{a}, \mathrm{b}}$ & $22.47 \pm 4.09$
\end{tabular}

Values are expressed as the mean \pm standard error. pCS was defined as the percentage $(\%)$ of positive calcified surface from the total examined area. P-values were calculated by the Mann-Whitney U test. ${ }^{\mathrm{a}, \mathrm{b}} \mathrm{P}<0.05,{ }^{\mathrm{c}, \mathrm{d}} \mathrm{P}<0.01$, e,f,g $\mathrm{P}<0.001$. pCS, pathological calcification score; pSFA, proximal third of the superficial femoral artery; dSFA, distal segment of the superficial femoral artery; PA, popliteal artery; CA, carotid artery.

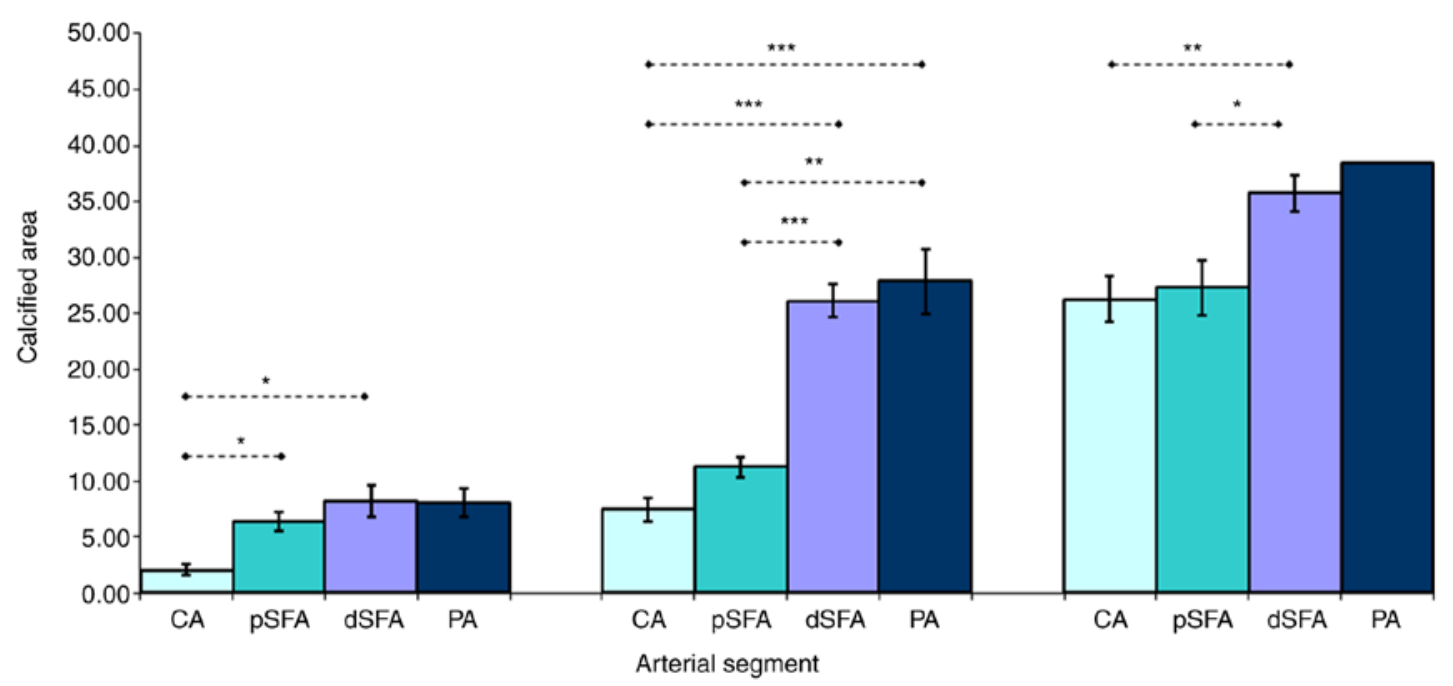

Figure 5. Bar graph showing the representation of calcification types in different arterial segments. Statistically significant differences are marked with an asterisk $\left({ }^{*} \mathrm{P}<0.05,{ }^{* * *} \mathrm{P}<0.01,{ }^{* * * *} \mathrm{P}<0.001\right)$. CA, carotid artery, pSFA, proximal third of the superficial femoral artery; dSFA, distal segment of the superficial femoral artery; PA, popliteal artery.

arterial disease (PAD) and critical limb ischemia (CLI), the true prevalence of vascular calcification in symptomatic PAD patients remains undefined. Regarding plaque histology, in our comparative study, we observed that femoral and carotid plaques showed different morphology. The establishment of the histological grade of the atherosclerotic plaques in the examined arterial segments showed that high grade American Heart Association (AHA) categories develop predominantly at the level of the superficial femoral artery (SFA) compared to the CA. This site-dependent pattern of atherosclerotic plaques is probably the result of the structure of the vascular wall and of differences in hemodynamics. The pathophysiological studies underlined that artery type-dependent plaque features result from the complex effect of blood flow, represented by peak/mean wall shear rates (higher in CA than FPA) (27) and variations in the speed of blood circulation, that influence the residence time with endothelial cells of lipoproteins, inflammatory cells and molecules which have an important role in the mechanism of atherosclerosis (28). Another finding of our study refers to calcification. Different femoral artery segments were more susceptible to calcification in comparison with the CA. Vascular calcification occurs in all atherosclerotic lesions independently of their arterial location, but in peripheral arteries differs quantitatively. Compared to the CA atherosclerosis, the FPA exhibited increased calcification (29). At the same time the location of calcification in plaque structures also plays an important role in plaque progression. In advanced plaques, even small calcifications of the thin fibrous cap may lead to plaque destabilization and rupture. By contrast, large calcification areas in the intimal-medial boundary do not contribute to lesion instability, but may be involved in the development of stable atherosclerotic disease (30).

Represented by a relatively equal number, we initially compared the atherosclerotic plaque structures in two arterial segment, the bifurcation of CA and proximal (p)SFA arteries with similar morphology regarding the wall structure (both being transitional type between the elastic and the muscular artery) (31). Despite the fact that the thickness of the carotid and femoral atherosclerotic plaques did not differ in the two localizations, we found differences in terms of nodular and extensive calcification types in favor of the pSFA plaques. Regarding nodular calcification, similar results have been previously noted by Herisson et al (18), but they did not sufficiently characterize the ratio between the mineral mass and 
the surrounding environments around the calcification within plaques. Based on the findings in the literature according to which superficial calcifications are independently associated with plaque vulnerability, and the type of calcification extension (dispersed or compacted), dimensions, shapes and positions may play different roles in plaque evolution (11), we continued our study by determining the total calcified surface area (as pCS) of remote plaques by morphometry and we focused on the extent of calcification in terms of spectrum between the sheet-like nodular-extensive calcification. In the present study we showed that the calcified area varied depending on the arterial segment affected by atherosclerosis; the total calcified area was higher in femoral plaques than in carotid plaques, but without a significant difference between the plaques of the two arteries with the same structure (pSFA and CA). At the same time, we noted a significant increase in mineralized surface at the level of the different FPA segments in parallel with a decrease in the vascular lumen diameter. Differences in the total calcification of plaques depending on the involved arterial segments were consistent with the histological grade, according to which most of the femoral plaques were classified as fibrocalcific (VII and VIII AHA types), whereas carotid plaques were classified as fibrous cap atheroma (IV and V types) (18).

At the same time, we found that, even when the three femoral segment plaques and carotid plaques explain different calcification pattern, the total mineralized area did not depend on the shape and pattern of the plaques, but rather on vascular caliber; in parallel to the narrowing of the lumen, the calcified area of the plaque increased. This finding was also supported by the results obtained in the case of an extensive calcification pattern, in the sense that the mineralized area did not differ between the CA and pSFA plaques, but in contrast, the pCS of the CA plaques was lower than those measured in distal (d)SFA.

Although several molecular aspects of this mechanism have been elucidated regarding the two types of arterial calcification in the different vessel types (large elastic vs. smaller muscular arteries) and parts (proximal vs. distal) $(32,33)$, few morphologic studies have focused on the plaque calcification in the different segments of the FPA in comparison with the CA. The most significant results are in the study of Herisson et al (18), who focused on the calcification pattern, complemented with quantitative measurements of calcium and lipids within the plaque. This complex approach of calcification underscored the high prevalence of sheet-like and nodular calcification parallel with significantly higher amounts of calcium at the level of FA plaques in comparison with CA plaques, and did not lead to the staging of calcification according to different segments of AFP.

In studies that included the measurement of calcium scores and occlusion grade, it was reported that patients with PAD presenting increased occlusion and calcification scores were strongly associated with more severe stages of ischemia (34), and independently predicted amputation and mortality (35-37). However, the exact mechanism of atherosclerotic calcification, including the territorial distribution and the size of calcified regions within atherosclerotic lesions and its role in plaque vulnerability, remains incompletely understood (21).

Microcalcifications $(0.5-50 \mathrm{~mm})$ represent an early stage in the spectrum of the vascular calcification cascade. They are considered predictors of cardiovascular events, leading to rupture of the plaque through the transfer of the stress from the interface between the fibrous cap and lipid core to the interface between the fibrous cap and vessel lumen (38). Some authors claim that although extensive calcifications are associated with plaque stability, the role of the calcification size in plaque vulnerability may be biphasic. They explain this phenomenon by the interaction of mechanical forces with the mass of the plaque. Normally, mechanical stress is expected to be concentrated at the interfaces between materials with different stiffness, within a plaque at the interfaces between calcium deposits and the rest of the plaque elements. Calcium stiffness is at least four times higher than that of other plaque components (39), as a result, if the degree of calcification increases, the calcium deposits merge and the soft surface area progressively decreases (11).

We are aware of the limitations of our study. The relatively small sample number of a single center material characterizes the CA and FPA plaques at a well-determined moment. The morphological aspects of the plaques of the two arteries from different patients compromise the comparison due to their development in different pathological conditions. At the same time, most of the lesions came from advanced atherosclerotic plaques, with extensive calcification associated with a relatively small number of sheet-like calcifications. Regarding the morphometric analysis, we determined the total surface and not the total amount of mineral salts of the plaques, because different patterns were combined in a single plaque, but we agree that the nodular type of macrocalcifications has a large volume but a small surface area $(40,41)$. A technical disadvantage of the study was the loss of calcification (during cutting) in some sections and morphology distortion. In some cases, we tried to overcome this by following the perimeter of the calcified cavity, a method that is possible only in nodular calcifications.

In conclusion, despite being exposed to similar risk factors, peripheral arteries develop heterogeneous lesions. Femoral and carotid plaques show different morphologies and tendencies for calcification. In the present study, we demonstrated that a population with similar demographic and biological data develops at the level of smaller caliber arteries (femoral-popliteal/popliteal artery) larger areas of calcification than at the level of larger caliber arteries (femoral/carotid). These results suggest that the mechanism is site-specific, and wall structure-dependent. In advanced carotid and FPA atherosclerosis, calcification has a heterogeneous pattern with a simultaneous presence of large calcified area and numerous small calcific patches. Due to the heterogeneous composition of the calcified plaques, extensive calcification remains controversial in terms of plaque stability.

\section{Acknowledgements}

Not applicable.

\section{Funding}

No funding was received.

\section{Availability of data and materials}

The data and materials that support the results of this study are available from the corresponding author $(\mathrm{EH})$. 


\section{Authors' contributions}

MCC conceived the study, coordinated the surgical team, performed the surgery, analyzed and interpreted the patient data. MCC, ER and CM selected the patients and performed the surgery. EH designed the study, performed histopathological and morphometric analyses of the biopsies, contributed to data analysis, performed figure design and provided critical review of the manuscript and was responsible for the final edited version. GBM performed the histopathological and morphometric analyses of the biopsies, contributed to data analysis and was responsible for the acquisition of the data. VAM acquired the data and revised the manuscript critically for important intellectual content and coordinated the research. All authors contributed to data interpretation and editing the manuscript and read and approved the final version.

\section{Ethics approval and consent to participate}

The study was approved by the Ethics Committee of 'George Emil Palade' University of Medicine, Pharmacy, Science and Technology of Târgu-Mureș (no. 884 and 11420/30.04). All patients provided informed consent for inclusion in the study.

\section{Patient consent for publication}

This manuscript does not contain particular cases, personal information or images which would require patient personal consent.

\section{Competing interests}

The authors declare that they have no competing interests.

\section{References}

1. Eurostat:YourkeytoEuropeanstatistics:https://ec.europa.eu/eurostat/ statistics-xplained/index.php/Causes_of_death_statistics_-people_ over_65https://ec.europa.eu/eurostat/statistics-explained/index.php/ Cardiovascular_diseases_statistics.

2. Singh RB, Mengi SA, Xu YJ, Arneja AS and Dhalla NS: Pathogenesis of atherosclerosis: A multifactorial process. Exp Clin Cardiol 7: 40-53, 2002.

3. Albanese I, Khan K, Barratt B, Al-Kindi H and Schwertani A: Atherosclerotic calcification: Wnt is the hint. J Am Heart Assoc 7: e007356, 2018.

4. Peace A, van Mil A, Jones H and Thijssen DHJ: Similarities and differences between carotid artery and coronary artery function. Curr Cardiol Rev 14: 254-263, 2018.

5. Schiano V, Sirico G, Giugliano G, Laurenzano E, Brevetti L, Perrino C, Brevetti G and Esposito G: Femoral plaque echogenicity and cardiovascular risk in claudicants. JACC Cardiovasc Imaging 5: 348-357, 2012.

6. Golomb BA, Dang TT and Criqui MH: Peripheral arterial disease: Morbidity and mortality implications. Circulation 114 688-699, 2006

7. Maleckis K, Anttila E, Aylward P, Poulson W, Desyatova A, MacTaggart J and Kamenskiy A: Nitinol Stents in the femoropopliteal artery: A mechanical perspective on material, design, and performance. Ann Biomed Eng 46: 684-704, 2018.

8. Kwee RM: Systematic review on the association between calcification in carotid plaques and clinical ischemic symptoms. J Vasc Surg 51: 1015-1025, 2010.

9. Shioi A and Ikari Y: Plaque Calcification during atherosclerosis progression and regression. J Atheroscler Thromb 25: 294-303, 2018.

10. Yang J, Pan X, Zhang B, Yan Y, Huang Y, Woolf AK, Gillard JH, Teng $Z$ and Hui P: Superficial and multiple calcifications and ulceration associate with intraplaque hemorrhage in the carotid atherosclerotic plaque. Eur Radiol 28: 4968-4977, 2018.
11. Shi X, Gao J, Lv Q, Cai H, Wang F, Ye R and Liu X: Calcification in atherosclerotic plaque vulnerability: Friend or Foe? Front Physiol 11: 56, 2020.

12. Tang L, Cui QW, Liu DP and Fu YY: The number of stents was an independent risk of stent restenosis in patients undergoing percutaneous coronary intervention. Medicine (Baltimore) 98: e18312, 2019.

13. Laird JR and Yeo KK: The treatment of femoropopliteal in-stent restenosis: Back to the future. J Am Coll Cardiol 59: 24-25, 2012.

14. Gerardi D, Alfani A, Tesorio T, Cioppa A, Esposito G and Stabile E: Drug-coated balloon in superficial femoral artery in-stent restenosis. Postepy Kardiol Interwencyjnej 14: 9-14, 2018.

15. Gaudry M, Bartoli JM, Bal L, Giorgi R, De Masi M, Magnan PE and Piquet P: Anatomical and technical factors influence the rate of in-stent restenosis following carotid artery stenting for the treatment of post-carotid endarterectomy stenosis. PLoS One 11: e0161716, 2016.

16. Katano H, Nishikawa Y, Yamada $\mathrm{H}$ and Mase M: Calcification in original plaque and restenosis following carotid artery stenting. Surg Neurol Int 8: 279, 2017.

17. Katano H, Mase M, Nishikawa Y, Yamada $\mathrm{H}$ and Yamada $\mathrm{K}$ : Analysis of recurrent stenosis after carotid endarterectomy featuring primary plaque calcification. Neurosurgery $80: 863-70$, 2017.

18. Herisson F, Heymann MF, Chetiveaux M, Charrier C, Battaglia S, Pilet P, Rouillon T, Krempf M, Lemarchand P, Heymann D and Gouëffic Y: Carotid and femoral atherosclerotic plaques show different morphology. Atherosclerosis 216: 348-354, 2011.

19. Kelly-Arnold A, Maldonado N, Laudier D, Aikawa E, Cardoso L and Weinbaum S: Revised microcalcification hypothesis for fibrous cap rupture in human coronary arteries. PNAS 110: 10741-1046, 2013.

20. Jinnouchi H, Sato Y, Sakamoto A, Cornelissen A, Mori M, Kawakami R, Gadhoke NV, Kolodgie FD, Virmani R and Finn AV: Calcium deposition within coronary atherosclerotic lesion: Implications for plaque stability. Atherosclerosis 306: 85-95, 2020.

21. Han RI, Wheeler TM, Lumsden AB, Reardon MJ, Lawrie GM, Grande-Allen KJ, Morrisett JD and Brunner G: Morphometric analysis of calcification and fibrous layer thickness in carotid endarterectomy tissues. Comput Biol Med 70: 210-219, 2016.

22. Tavakoli $\mathrm{S}$ and Sadeghi MM: ${ }^{18} \mathrm{~F}-\mathrm{NaF}$ PET and plaque calcification: How complicated can it be? Circ Cardiovasc Imaging 12: e008712, 2019.

23. Stary HC: Natural history and histological classification of atherosclerotic lesion: An update. Arterioscler Thromb Vasc Biol 20: 1177-1178, 2000.

24. Ferreira $T$ and Rasband $W$ : ImageJ User Guide. Image Processing and Analysis in Java. National Institutes of Health, 2012. http://rsb.info.nih.gov/ij.

25. Redgrave JNE, Lovett JK, Gallagher PJ and Rothwell P: Histological assessment of 526 symptomatic carotid plaques in relation to the nature and timing of ischemic symptoms: The Oxford plaque study. Circulation 113: 2320-2328, 2006.

26. Zhu G, Hom J, Li Y, Jiang B, Rodriguez F, Fleischmann D, Saloner D, Porcu M, Zhang Y, Saba L and Wintermark M: Carotid plaque imaging and the risk of atherosclerotic cardiovascular disease. Cardiovasc Diagn Ther 10: 1048-1067, 2020.

27. Reneman RS, Arts T and Hoeks AP: Wall shear stress-an important determinant of endothelial cell function and structure-in the arterial system in vivo discrepancies with theory. J Vasc Res 43: 251-269, 2006.

28. VanderLaan PA, Reardon CA and Getz GS: Site specificity of atherosclerosis: Site selective responses to atherosclerotic modulators. Arterioscler Thromb Vasc Biol 24: 12-22, 2004.

29. Otsuka F, Sakakura K, Yahagi K, Joner M and Virmani R: Has our understanding of calcification in human coronary atherosclerosis progressed? Arterioscler Thromb Vasc Biol 34: 724-736, 2014.

30. Chistiakov DA, Myasoedova VA, Melnichenko AA, Grechko AV and Orekhov AN: Calcifying matrix vesicles and atherosclerosis. Biomed Res Int 2017: 7463590, 2017.

31. Janzen J: The microscopic transitional zone between elastic and muscular arteries. Arch Mal Coeur Vaiss 97: 909-914, 2004.

32. Amann K: Media calcification and intima calcification are distinct entities in chronic kidney disease. Clin J Am Soc Nephrol 3: 1599-1605, 2008.

33. Allison MA, His S, Wassel CL, Morgan C, Ix JH, Wright CM and Criqui $\mathrm{MH}$ : Calcified atherosclerosis in different vascular beds and the risk of mortality. Arterioscler Thromb Vasc Biol 32: 140-146, 2012 
34. Zettervall SL, Marshall AP,Fleser P and Guzman RJ: Association of arterial calcification with chronic limb ischemia in patients with peripheral artery disease. J Vasc Surg 67: 507-513, 2018.

35. Huang CL, Wu IH, Wu YW, Hwang JJ, Wang SS, Chen WJ, Lee WJ and Yang WS: Association of lower extremity arterial calcification with amputation and mortality in patients with symptomatic peripheral artery disease. PLoS One 9: e90201, 2014.

36. Blacher J, Guerin AP, Pannier B, Marchais SJ and London GM: Arterial calcifications, arterial stiffness, and cardiovascular risk in end-stage renal disease. Hypertension 38: 938-942, 2001.

37. Guzman RJ, Brinkley DM, Schumacher PM, Donahue RMJ, Beavers H and Qin X: Tibial artery calcification as a marker of amputation risk in patients with peripheral arterial disease. J Am Coll Cardiol 51: 1967-1974, 2008.

38. Rambhia SH, Liang X, Xenos M, Alemu Y, Maldonado N, Kelly A, Chakraborti S, Weinbaum S, Cardoso L, Einav S and Bluestein D: Microcalcifications increase coronary vulnerable plaque rupturepotential: A patient-based micro-CT fluid-structure interaction study. Ann Biomed Eng 40: 1443-1454, 2012.
39. Lee RT, Grodzinsky AJ, Frank EH, Kamm RD and Schoen FJ: Structure-dependent dynamic mechanical behavior of fibrous caps from human atherosclerotic plaques. Circulation 83: 1764-1770, 1993

40. Wang Y, Osborne MT, Tung B, Li M and Li Y: Imaging cardiovascular calcification. J Am Heart Assoc 7: e008564, 2018.

41. Dweck MR, Aikawa E, Newby DE, Tarkin JM, Rudd JH, Narula $\mathbf{J}$ and Fayad ZA: Noninvasive molecular imaging of disease activity in atherosclerosis. Circ Res 119: 330-340, 2016.

c) (i) $\Theta$ This work is licensed under a Creative Common Attribution-NonCommercial-NoDerivatives 4.0 International (CC BY-NC-ND 4.0) License. 